\title{
Stearoyl-CoA desaturase-1 is a key factor for lung cancer-initiating cells
}

\author{
A Noto ${ }^{1,2}$, S Raffa ${ }^{1,3,4}$, C De Vitis ${ }^{2,5}$, G Roscillii, ${ }^{1,6}$, Malpicci ${ }^{2}$, P Coluccia ${ }^{2}$, A Di Napoli ${ }^{1,3}$, A Ricci ${ }^{1,3}$, MR Giovagnoli ${ }^{1,3}$, L Aurisicchio ${ }^{6,7}$, \\ MR Torrisi ${ }^{1,3,4}$, G Ciliberto $^{\star, 8}$ and $R$ Mancini ${ }^{1,2}$
}

In recent years, studies of cancer development and recurrence have been influenced by the cancer stem cells (CSCs)/cancerinitiating cells (CICs) hypothesis. According to this, cancer is sustained by highly positioned, chemoresistant cells with extensive capacity of self renewal, which are responsible for disease relapse after chemotherapy. Growth of cancer cells as three-dimensional non-adherent spheroids is regarded as a useful methodology to enrich for cells endowed with CSC-like features. We have recently reported that cell cultures derived from malignant pleural effusions (MPEs) of patients affected by adenocarcinoma of the lung are able to efficiently form spheroids in non-adherent conditions supplemented with growth factors. By expression profiling, we were able to identify a set of genes whose expression is significantly upregulated in lung tumor spheroids versus adherent cultures. One of the most strongly upregulated gene was stearoyl-CoA desaturase (SCD1), the main enzyme responsible for the conversion of saturated into monounsaturated fatty acids. In the present study, we show both by RNA interference and through the use of a small molecule inhibitor that SCD1 is required for lung cancer spheroids propagation both in stable cell lines and in MPE-derived primary tumor cultures. Morphological examination and image analysis of the tumor spheroids formed in the presence of SCD1 inhibitors showed a different pattern of growth characterized by irregular cell aggregates. Electron microscopy revealed that the treated spheroids displayed several features of cellular damage and immunofluorescence analysis on optical serial sections showed apoptotic cells positive for the M30 marker, most of them positive also for the stemness marker ALDH1A1, thus suggesting that the SCD1 inhibitor is selectively killing cells with stem-like properties. Furthermore, SCD1-inhibited lung cancer cells were strongly impaired in their in vivo tumorigenicity and ALDH1A1 expression. These results suggest that SCD1 is a critical target in lung cancer tumor-initiating cells.

Cell Death and Disease (2013) 4, e947; doi:10.1038/cddis.2013.444; published online 5 December 2013

Subject Category: Cancer

Metabolic deregulation has emerged as one of the main features of cancer cells. Tumor cells display a radical modification of cellular metabolism, with increased glycolysis and lipogenesis, to support rapid growth and proliferation. ${ }^{1}$ A distinctive aspect of the lipogenic transformation is that cancer cells contain a large pool of monounsaturated fatty acids (MUFAs). MUFAs represent the precursors of phospholipids, tryglicerides, cholesterol esters, diacylglycerols and wax esters, which are the main component of membranes. Changes in lipid membrane composition can affect membrane fluidity, signaling and, consequently, gene expression. ${ }^{2,3}$

MUFAs are generated from saturated fatty acids by the action of steroyl Co-A desaturases. Two SCD isoforms (SCD1 and 5) have been identified in humans and exhibit different tissue distribution patterns but share the same enzymatic function. SCD1 is found in almost all tissues with a major expression in liver, whereas SCD5 expression is restricted to pancreas and brain. Of these isoforms, SCD1 is the predominant one and is expressed ubiquitously among tissues. $^{4-7}$ Recent evidences suggest that SCD1 has a supporting role in many human cancers including lung, breast, prostate and clear cell renal cell carcinoma. ${ }^{8-16}$ It has been reported that in lung cancer SCD1 contributes to maintain a shift in lipid metabolism (increase in lipogenesis and inhibition of fatty acid oxidation) and intracellular signaling (activation of Akt signals and deactivation of the AMPK pathway), therefore favoring an accelerated rate of cell proliferation, increased invasiveness, enhanced survival and, ultimately, a greater tumorigenic capacity. ${ }^{8,15}$ The growing evidences in support of SCD1 as a cancer target opens the possibility to utilize recently generated small molecule inhibitors of SCD1 activity as anticancer tools. ${ }^{17,18}$

A new perspective to our understanding of how cancer develops and recurs after initial therapy, and a further

\footnotetext{
'Department of Clinical and Molecular Medicine, Sapienza University of Rome, Italy; ' 2 Laboratory of Research and Diagnostics, Department of Surgery 'P.Valdoni', Sapienza University of Rome; ${ }^{3}$ Azienda Ospedaliera S. Andrea, Rome, Italy; ${ }^{4}$ Institute Pasteur-Fondazione Cenci Bolognetti, Italy; ${ }^{5}$ Department of Experimental and Clinical Medicine, University of Catanzaro 'Magna Graecia', Italy; ${ }^{6}$ Takis srl, Via di Castel Romano, Rome, Italy; ${ }^{7}$ Biogem s.c a r.l., Ariano Irpino (AV), Italy and ${ }^{8}$ IRCCS Istituto Nazionale Tumori, Fondazione 'G. Pascale', Napoli, Italy

${ }^{*}$ Corresponding author: G Ciliberto, Scientific Directorate, Istituto Nazionale Tumori, Via Mariano Semmola, Napoli 80131, Italy. Tel: +390815903756; Fax: +390815461688; E-mail: g.ciliberto@ istitutotumori.na.it

Keywords: lung cancer; tumor spheroids; cancer stem cells; SCD1 inhibition; anoikis

Abbreviations: SCD1, stearoyl-CoA desaturase-1; MUFA, monounsaturated fatty acid; CIC, cancer-initiating cell; NSCLC, non-small cell lung cancer; MPE, malignant pleural effusion; ALDH1A1, aldehyde dehydrogenase $1 \mathrm{~A} 1$

Received 13.9.13; revised 04.10.13; accepted 08.10.13; Edited by G Raschellá
} 
opportunity to identify therapeutic agents with a new mechanism of action has been opened by the cancer stem cell hypothesis. According to this theory, cancer is sustained by a population of cells with stem cell-like properties whose distinctive feature is their extensive capacity to self-renew, and to produce progenitor cells and terminally differentiated progeny. ${ }^{19-22}$ The putative CSCs are themselves mostly quiescent for proliferation, are resistant to chemotherapeutic agents and therefore are thought to be responsible for disease relapse and for the emergence of resistance to therapies. ${ }^{23-25}$ The identification of CSCs remains challenging and several CSC markers have been proposed. Among them, ALDH1A1 expression and its enzymatic activity seems to best correlate with the presence of CSCs and the aggressiveness of lung tumors. ${ }^{26-29}$ Although recent technological advances in the isolation and characterization of CSCs have led to a better understanding of their biology, our knowledge of the key factors responsible for their survival and propagation is still limited.

In a previous study, from our group primary tumor cultures obtained from malignant pleural effusions (MPEs) of patients with adenocarcinoma of the lung were characterized for the presence of cells with ALDH1A1 activity. We showed that in the majority of the samples analyzed, the percentage of ALDH1A1 bright cells increased on culturing in spheroid conditions, suggesting the presence of putative CSCs in MPE-derived primary cultures (MPEDCC) and their enrichment in cancer spheroids. Most importantly gene expression profiling of spheroids versus adherent cultures allowed the identification of a gene expression signature of spheroids composed by a limited set of co-regulated genes. Among them, one of the most significantly upregulated gene in spheroids was SCD1. ${ }^{30}$

The goal of the present study was to investigate the expression and functional significance of SCD1 in lung cancer cell cultures. For this purpose, we studied the cellular response of tumor spheroids generated from either a stabilized lung cancer cell line or from MPE-derived primary cultures to SCD1 inhibition. This was achieved with the use of the small molecule inhibitor MF-438 ${ }^{17,18}$ or by RNA interference. The results demonstrate that SCD1 may represent a key therapeutic target for lung cancer stem cells.

\section{Results}

SCD1 upregulation in lung cancer spheroids goes in parallel with increased ALDH1A1 activity. Lung cancer spheroids obtained from MPE-derived cell cultures (MPEDCC) could be serially propagated in vitro and gave rise efficiently to tumor masses reproducing the same histopathological features of the original human tumors when implanted in immunodeficient mice. ${ }^{30,31}$ Gene expression analysis led to the identification of a set of 19 genes overexpressed in tumor spheroids versus adherent cultures. Among the overexpressed genes, SCD1 was one of the most significantly upregulated in spheroids. ${ }^{30}$

We first determined the spheroid-forming efficiency in a panel of five MPE-derived cell cultures named $P e d / 10$, $\mathrm{Pe}$ e/10, $\mathrm{Pe}$ o/11, $\mathrm{Pe} \mathrm{p} / 11$ and $\mathrm{Pe} \mathrm{s} / 11$ obtained from distinct non-small cell lung cancer (NSCLC) patients (for their origin and nomenclature see the Materials and Methods section and Mancini et $\mathrm{al}^{30}$ ) and in a stable NSCLC cell line (NCI-H460) trying to establish a correlation with ALDH1A1 activity. The results, shown in Figures $1 a$ and $b$, demonstrate that cells with higher ALDH1A1 activity show the highest efficiency in tumor spheroid formation. This is clearly evident when comparing the behavior of $\mathrm{NCl}-\mathrm{H} 460, \mathrm{Pe} \mathrm{o} / 11, \mathrm{Pe} \mathrm{d} / 10$ and $\mathrm{Pe} e / 10$, which have ALDH1A1 positive cells ranging from $25.1 \%$ to $11.5 \%$ and a strong spheroid-forming ability, with that of $\mathrm{Pe} \mathrm{s} /$ 11 and $\mathrm{Pe} p / 11$, where a value for ALDH1A1 activity below $5 \%$, was accompanied by a reduced ability to form spheroids (Figures $1 \mathrm{a}$ and $\mathrm{b}$ ).

Next, to confirm and expand our previous microarray data, we verified by western blotting and real-time RT-PCR, SCD1 expression in three of these selected primary cultures and in the stable cell line $\mathrm{NCl}-\mathrm{H} 460$. As shown in Figures 1c and d, all four lung cancer cultures analyzed presented a more than 10-fold increase of SCD1 mRNA levels in spheroids compared with adherent cells (Student's $t$-test: $P<0.01$ for $\mathrm{NCl}$ $\mathrm{H} 460, \mathrm{Pe}$ e/10 and $\mathrm{Pe} \mathrm{o} / 11 ; P<0.05$ for $\mathrm{Pe} \mathrm{d} / 10$ ), which was paralleled by a constant increase in SCD1 protein levels.

SCD1 is required for spheroid formation. On the basis of the previous data, we next assessed the contribution of SCD1 to the formation of spheroids. Cell cultures were transfected with a siRNA against SCD1 or a control siRNA (scramble) and a spheroid-forming assay was performed. The results showed a dramatic reduction of the number of spheroids formed in SCD1-depleted cultures: siSCD1-transfected cell cultures show a 2- to 3-fold decrease of spheroidforming efficiency, compared with the cultures transfected with a control siRNA (Student's $t$-test: $P<0.01$ for $\mathrm{NCl}-\mathrm{H} 460$ and $\mathrm{Pe} \mathrm{o} / 11 ; P<0.05$ for $\mathrm{Pe} \mathrm{d} / 10$ ) (Figures $2 \mathrm{a}$ and $\mathrm{b}$ ).

MF-438, a thiadiazole-pyridazine derivate, was recently described as a potent, orally available SCD1 enzymatic inhibitor that exhibits good pharmacokinetics and metabolic stability. ${ }^{17,18}$ First, we tested in adherent cultures from MPEDCC and in the NCl-H460 cell line the effect of MF-438 on cell viability in a 72-h growth inhibition assay. All cell cultures analyzed were highly resistant to this inhibitor, showing $\mathrm{IC}_{50}$ values between 20 and $50 \mu \mathrm{M}$ (Supplementary Figure 1). To examine the effect of SCD1 inhibition on spheroid formation, the same inhibitor was tested in a spheroid-forming assay. Surprisingly, as shown in Figure 2c and Table 1, $\mathrm{Pe} \mathrm{o/11} \mathrm{as} \mathrm{well} \mathrm{as} \mathrm{NCl}-\mathrm{H} 460$ spheroids were very sensitive to $\mathrm{MF}-438$ with $\mathrm{IC}_{50}$ values below $1 \mu \mathrm{M}$, showing a more than 100 -fold sensitization compared with adherent cultures. To confirm the specificity of the inhibition, we performed rescue experiments using oleate, the main product of SCD1 activity, in spheroids treated or not with MF438. As shown in Figure 2d, oleate supplementation in the form of conjugate with BSA (OA-BSA) partially but significantly reversed spheroids inhibition by MF-438, indicating that fatty acid desaturation by SCD1 is essential for spheroids formation (Student's $t$-test: $P<0.05$ ). Same results were also obtained using a different enzymatic inhibitor of SCD1 A $939572^{10}$ (Supplementary Figure 2).

We also analyzed the three-dimensional morphology of tumor spheroids grown in sphere medium with or without the 

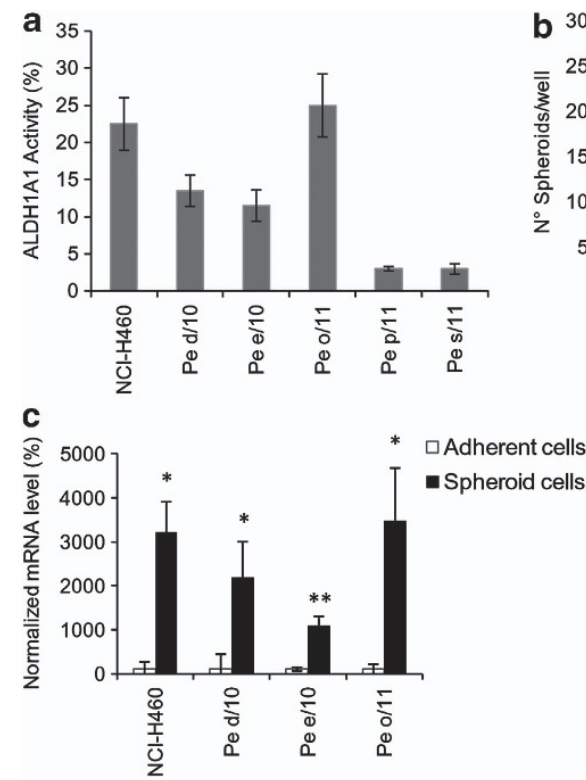

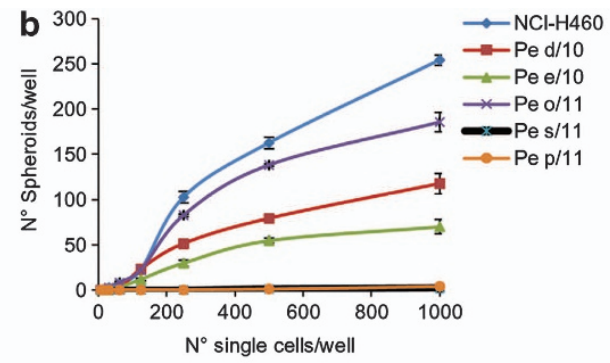

d

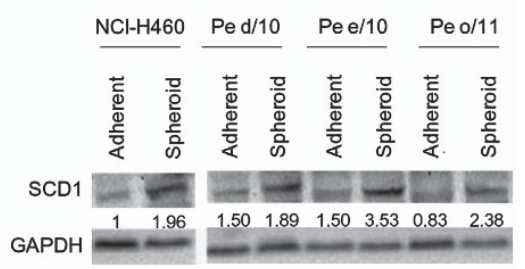

Figure 1 SCD1 is overexpressed in lung spheroid cultures. (a) ALDH1A1 activity was measured by flow cytometry, as described in Materials and Methods, in spheroid cultures obtained from five lung primary cell cultures and a stable cell line. Results indicated an increased ALDH1A1 activity in Pe d/10 (13.5\%), Pe e/10 (11.5\%), Pe 0/11 $(25.1 \%)$ and $\mathrm{NCl}-\mathrm{H} 460(22.5 \%)$ spheroids. Results represent the means \pm S.D. of three independent experiments. (b) Spheroid-forming ability was also evaluated using different cell density in serum-free medium supplemented with growth factors for 8 days. Increased spherogenicity was observed in spheroid cultures obtained from three primary cell cultures and in the stable cell line $\mathrm{NCl}-\mathrm{H} 460$, which also displayed increased ALDH1A1 activity. Results are the means of three independent experiments; bars indicate S.D. (c) SCD1 mRNA level was increased more than 10-fold in spheroids cultures compared with adherent cultures. Results represented in the histogram are the means \pm S.D. of three independent experiments. GAPDH was used as housekeeping gene. Student's $t$-test ${ }^{*} P<0.01$ and ${ }^{* *} P<0.05$ in adherent versus spheroid cell cultures. (d) Representative western blotting showing SCD1 protein expression upregulation in three primary cultures and the stable cell line $\mathrm{NCl}-\mathrm{H} 460$ grown as spheroid in respect to adherent cultures. GAPDH was used as loading control

MF-438 inhibitor, using a dose $(1 \mu \mathrm{M})$, which did not affect cell morphology in $2 \mathrm{D}$ adherent cultures (data not shown) and below their $\mathrm{IC}_{50}$ values (Table 1). Although untreated cultures displayed a typical spheroid pattern of growth, characterized by sphericity of the multicellular structures $(\phi=0.92)$ and high percentage of tight spheroids (exceeding 50\%) (Figures 3a and $\mathrm{b}$, Table 2), the amount of tight spheroids in the presence of the SCD1 inhibitor was drastically reduced (10.3\%; Chi square test: $P<0.0001)$ and the cultures were characterized by high prevalence of irregular cell aggregates $(62.8 \%$ ) of lower sphericity $(\phi=0.80)$ with respect to the untreated cultures (Figures $3 \mathrm{~b}$ and $\mathrm{c}$ ).

Taken together these data suggest that SCD1 inhibition has a more pronounced effect on cancer stem cells enriched cultures which organize themselves as three-dimensional (3D) structures, and therefore that this enzyme can be a target specific for cells with cancer-initiating properties.

SCD1 impairment reduces expression of stem cell markers in spheroids. Next, we decided to assess the effect of SCD1 inhibition on the activity of ALDH1A1 enzyme. We generated spheroids in the presence of a siRNA specific for SCD1 and after $72 \mathrm{~h}$, we evaluated ALDH1A1 activity by flow cytometry. Both in $\mathrm{NCl}-\mathrm{H} 460$ and primary culture $\mathrm{Pe} \mathrm{o} / 11$, silencing of SCD1 induced a dramatic reduction of ALDH1A1-positive cells (ALDH bright) compared with control siRNA-transfected cells $(21.22 \%$ versus $10.68 \%$ in $\mathrm{NCl}-\mathrm{H} 460$ and $26.18 \%$ versus $11.86 \%$ in $\mathrm{Pe} 0 / 11$; Student's t-test: $P<0.05)$ (Figure $4 a)$.
We previously showed that MPEDCC undergo increased expression of stem cell markers Nanog and Oct4, when grown as spheroids. ${ }^{31}$ Therefore, we analyzed the effect of SCD1 downregulation during spheroid formation on the expression of these markers. Indeed, as shown in Figure $4 \mathrm{~b}$ both OCT4 and Nanog, mRNA levels were reduced $\sim 10$-fold in SCD1-depleted NCl-H460 spheroids (Student's $t$-test: $P<0.01)$.

A similar effect was also obtained using MF-438 in $\mathrm{Pe} 0 / 11$ and $\mathrm{NCl}-\mathrm{H} 460$ spheroids cultures. Untreated spheroids showed an elevated ALDH1A1 activity (22.34\% in $\mathrm{NCl}-$ $\mathrm{H} 460,28.66 \%$ in $\mathrm{Pe} \mathrm{o} / 11$ ) as compared with MF-438-treated spheroids $(10.11 \%$ in $\mathrm{NCl}-\mathrm{H} 460,12.76 \%$ in $\mathrm{Pe} \mathrm{o} / 11$; Student's t-test: $P<0.05$ ) (Figure $4 \mathrm{c}$ ). Importantly, spheroids formed in the presence of the inhibitor dramatically reduced their ALDH1A1 activity, which was restored by oleate addition (Supplementary Figure 3).

SCD1 inhibition induces cellular damage and anoikis in ALDH1A1-positive cells. The ability of cells to grow in conditions of non-adherence is linked to their resistance to anoikis (detachment-induced apoptosis). We previously reported that MPEDCC are resistant to anoikis when grown as 3D cultures and that this is partly dependent on the activation of the BDNF/TrkB axis. ${ }^{31}$ Hence, to determine the cellular response after treatment with MF-438, we carried out on $\mathrm{Pe} \mathrm{0} / 11$, a spheroid-forming assay in the presence of the inhibitor and monitored anoikis induction by flow cytometry using Annexin V. As shown in Figure 5a, 


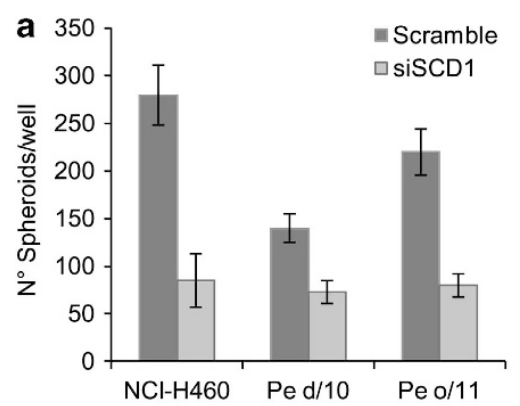

C

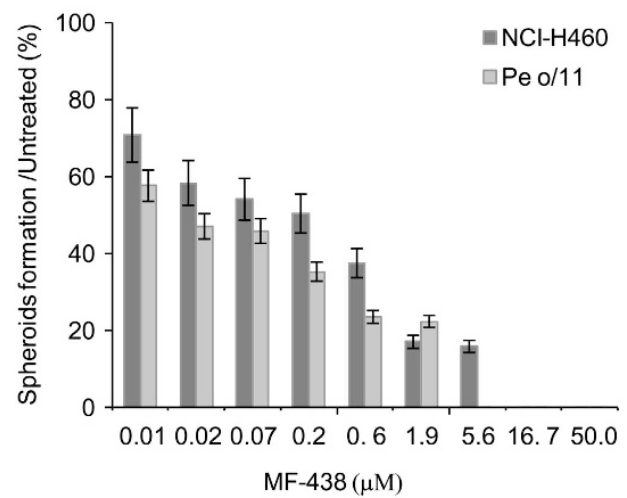

b

GAPDH
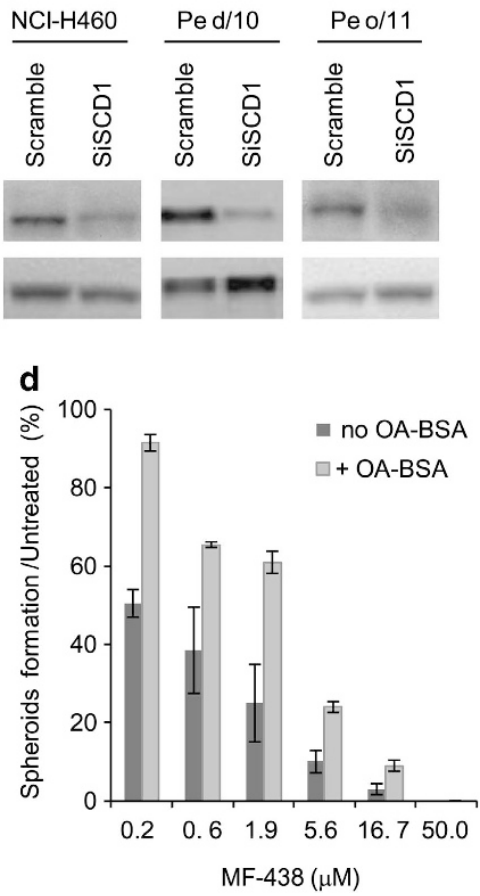

Figure 2 Silencing and pharmacological inhibition of SCD1 reduces the capability to form spheroids. (a) Spheroid-forming ability was reduced in NCI-H460, Pe d/10, Pe o/11 cell cultures after transfection with SCD1 siRNA, compared with control siRNA (scramble). Spheroids were allowed to form as described previously in Materials and methods, and were counted by optical microscopy. Results are the means \pm S.D. of three independent experiments. Student's $t$-test ${ }^{*} P<0.01$, ${ }^{\star \star} P<0.05$ in scramble versus siSCD1-transfected cells. (b) Representative western blotting of SCD1 protein expression was performed in siSCD1 and scramble-transfected cultures. GAPDH was used as normalization. (c) Spheroid-forming assay was carried out in NCl-H460 and Pe o/11 cell cultures treated with the indicated concentration of MF-438; results showed a decreased spheroid formation in the presence of increased concentration of the inhibitor. Results are the means of three independent experiments where bars indicate S.D. (d) A concentration of $1 \mu \mathrm{M}$ oleic acid-BSA can rescue the inhibitory effect of MF-438 when added to $\mathrm{NCl}-\mathrm{H} 460$ spheroids in a spheroid-forming assay. Data are means \pm S.D. of three independent experiments

Table 1 Spheroids cultures are more sensitive than adherent cultures to MF-438 inhibitor

\begin{tabular}{lccc}
\hline Cell line & $\begin{array}{c}\mathbf{I C}_{50} \\
\text { adherent } \\
\text { cells }\end{array}$ & $\begin{array}{c}\mathbf{I C}_{50} \\
\text { spheroid } \\
\text { cells }\end{array}$ & $\begin{array}{c}\text { Fold } \\
\text { sensitization }\end{array}$ \\
\hline $\mathrm{Pe} / 11$ & $40 \mu \mathrm{M}$ & $0.07 \mu \mathrm{M}$ & 571 \\
$\mathrm{NCl}-\mathrm{H} 460$ & $45 \mu \mathrm{M}$ & $0.28 \mu \mathrm{M}$ & 160 \\
\hline
\end{tabular}

$\mathrm{Pe}$ o/11 and $\mathrm{NCl}-\mathrm{H} 460$ adherent cultures were treated with MF-438 for $72 \mathrm{~h}$ and MTT assay was performed to evaluate cell viability. Spheroid-forming assay was performed in $\mathrm{NCl}-\mathrm{H} 460$ and $\mathrm{Pe}$ o/11 treated or not with increasing doses of MF-438. Data are means of three independent experiments. Bars indicate S.D. $\mathrm{IC}_{50}$ values were calculated as described in Materials and methods and fold sensitization was determined

a striking increased of apoptotic cells was observed in MF438-treated spheroids (16.2\% versus $4.3 \%$; Student's $t$-test: $P<0.01)$.

Transmission electron microscopy revealed that the aberrant spheroids formed in the presence of the SCD1 inhibitor displayed several ultrastructural features of cellular damage, such as cytoplasmic vacuolization, mitochondrial swelling, apoptotic nuclei with areas of marginal, densestained chromatin and, although less frequently, nuclear fragmentation (Figure 5b).
Finally, we demonstrated by quantitative immunofluorescence that the anoikis-inducing effect of MF-438 was specific for ALDH1A1-positive cells. Indeed, immunofluorescence analysis performed using the M30 CytoDEATH Fluorescein monoclonal antibody, able to detect the caspase-cleaved cytokeratin 18, showed that the percentage of apoptotic cells positive for the M30 marker was much higher in spheroids treated with MF-438 than in those grown in basal conditions (47.3\% versus $2.4 \%$; Student's $t$-test: $P<0.0001$; Figure $5 \mathrm{c}$, upper panel). Co-immunostaining for $\mathrm{M} 30$ and for the stemness marker ALDH1A1 confirmed the very high percentage of ALDH1A1-positive cells (86.2\%) in untreated spheroids, whereas treatment with MF-438 induced a clear decrease of ALDH1A1-positive cells (66.8\%; Student's t-test: $P<0.05$; Figure $5 c$ lower panel). In addition, the double labeling approach revealed that most of the apoptotic M30positive cells were also positive for ALDH1A1 (31.6\% of $\mathrm{M} 30^{+} / \mathrm{AldhA}^{+}{ }^{+}$versus $3.0 \%$ of $\mathrm{M} 30^{+} / \mathrm{AldhA} 1^{-}$cells in $\mathrm{Pe}$ $\mathrm{O} / 11 \mathrm{MF}-438$ treated), thus suggesting that the SCD1 inhibitor is selectively killing cells with stem like properties.

SCD1-depleted tumor spheroids have a strongly reduced ability to grow in vivo. To assess the effect of SCD1 inhibition on the capability to form tumors in vivo, we decided to perform in vivo xenograft studies in CD1 mice 
a

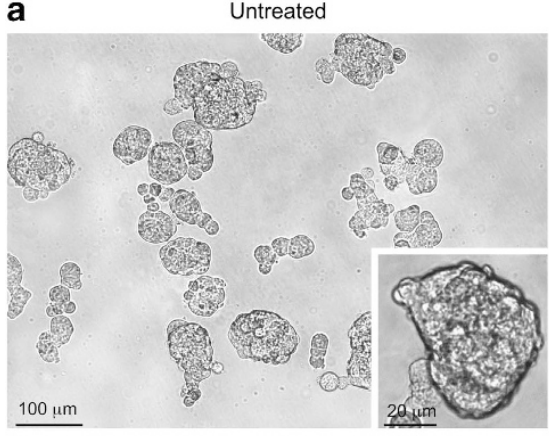

b

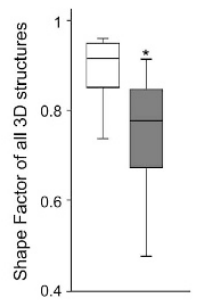

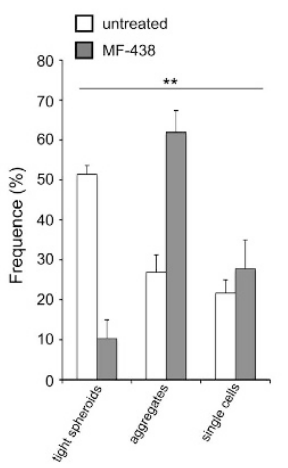

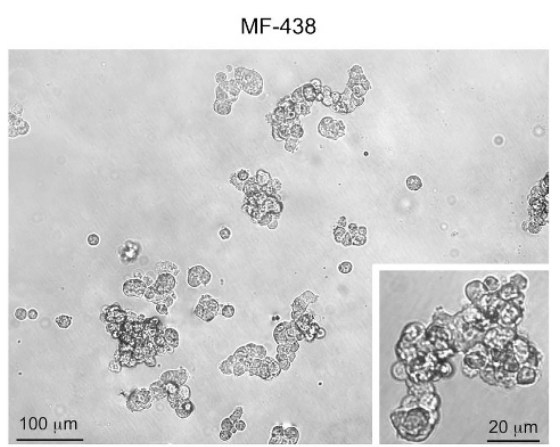

c

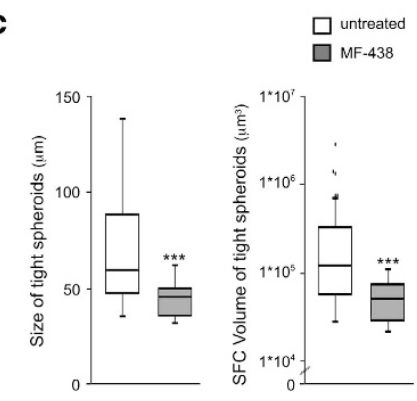

Figure 3 Three-dimensional and morphometric analysis of Pe o/11 tumor spheroids grown with or without SCD1 inhibitor. (a) Differential interference contrast microscopy of $\mathrm{Pe}$ o/11 cultures. The untreated Pe o/11 cultures (left panel) exhibited a typical spheroid pattern of growth with many densely packed spheres with indiscernible individual cells (see inset). The Pe o/11 cultures grown in presence of SCD1 inhibitor for $72 \mathrm{~h}$ were characterized by a high number of irregular multicellular structures (right panel) and displayed a small amount of tight spheroids. (b) Morphometric analysis of all multicellular structures from treated or untreated Pe o/11: the box-and-whisker plot of shape factor showed the higher sphericity of the tumor spheroids obtained from $\mathrm{Pe}$ o/11-untreated cultures. The central box represents the interquartile ranges, the middle line represents the median and the horizontal lines represent the minimum and the maximum value of observation range (Mann-Whitney test: ${ }^{*} P<0.0001$ ). At the morphological categorization of structures (see histogram), untreated Pe o/11 showed a higher prevalence of tight spheroids $(51.4 \%$ versus $10.3 \%)$ and a lower prevalence of irregular aggregated $(26.9 \%$ versus $62.8 \%$ ) compared with treated Pe o/11. Results reported in graph represent the mean values \pm S.E. of three experimental replicates (chi-square test: ${ }^{* \star} P<0.0001$ ). (c) Morphometric analysis of tight spheroids from treated or untreated $\mathrm{Pe}$ o/11: the box-and-whisker plots of size and SCF volume (see Materials and methods) showed that untreated cultures were characterized by a higher size and volume of tight spheroids respect to the treated cultures (Mann-Whitney test: ${ }^{* * *} P<0.001$ )

using the MPEDCC Pe o/11 and the stable NSCLC cell line $\mathrm{NCl}-\mathrm{H} 460$. For this purpose, spheroids were generated as described above in the absence or in the presence of a 48-h treatment with MF-438. Spheroids were then disaggregated, cells counted and an equivalent number of viable cells from SCD1-inhibited or normal cultures was injected subcutaneously in groups of 10 CD1 mice.

As reported before cells derived from normal $\mathrm{Pe} 0 / 11$ spheroids were more tumorigenic than an equivalent number of cells from adherent cultures (Figure 6a, Student's $t$-test, $P<0.05)$ probably as a consequence of the enriched number of ALDH1A1-expressing cells in the spheroid cultures. ${ }^{31}$ In contrast, cells derived from spheroids generated in the presence of MF-438 showed a strongly decreased tumorigenic potential (Figure 6a, Student's $t$-test: $P<0.05$ ). Different results were obtained for $\mathrm{NCl}-\mathrm{H} 460$ xenografts. In this case, the inhibitory effect of MF-438 was clearly evident in the percentage of tumor engraftment rather than in the size of the tumor masses generated (Supplementary Table 1 and data not shown).

Finally, we monitored ALDH1A1 expression in xenografts by immunostaining of paraffin sections. Interestingly, both $\mathrm{NCl}-\mathrm{H} 460$ and $\mathrm{Pe}$ o/11 xenografts obtained with cells generated from SCD1-inhibited spheroids showed a nearly complete lack of ALDH1A1 staining as compared with control tumors (Figure 6b).

\section{Discussion}

Lung cancer is the most common invasive cancer worldwide and includes a broad variety of histological subtypes, which belong to two main groups, small cell lung cancer (SCLC) and non-small cell lung cancer (NSCLC), which account approximately for $20 \%$ and $80 \%$ of all lung cancers cases, respectively. In spite of the introduction in the past decades of several targeted therapies, lung cancer remains a major health problem and the primary cause of deaths for cancer. ${ }^{32}$ The development of more effective therapies, which target central molecules involved in the progression and the proliferation of cancer cells, will be an efficient approach to treat lung cancer.

De novo lipogenesis seems to have a central role in the maintenance and progression of many human cancers. Cancer cells increase their lipid content to support growth, proliferation and survival. SCD1 is the central enzyme involved in the genesis of monounsatureted fatty acids from saturated fatty acids; MUFA and SFA are the most abundant fatty acids in cells, and the ratio SFA/MUFA can affect 
membrane fluidity and signaling. ${ }^{2,3}$ SCD1 is found to be overexpressed in many tumors and an increased number of evidences suggest that its enhanced activity promotes tumor growth. $^{8-16}$

In this study, we wanted to define the role of SCD1 in lung cancer using as study system spheroids derived from primary tumor cultures and from a stable cell line. Spheroid cultures overexpress markers of staminality, are more resistant to chemotherapies and are able to switch from an epithelial to a mesenchymal phenotype. ${ }^{33}$ In this paper, we first demonstrated that not all NSCLC cell cultures are able to form tight spheroids when cultured in spheroid medium, and that this ability correlates with the level of activity of the CSC-like marker ALDH1A1. ${ }^{26-29}$ The expression of SCD1 was assessed in spheroid cultures, which had an increased ALDH1A1 activity, compared with adherent 2D cultures which, on the contrary, showed a decreased ALDH1A1 activity (data not shown and Mancini et al..$^{30}$ and Ricci et $a{ }^{31}{ }^{31}$ ). We determined a strong upregulation of SCD1 at the protein and RNA levels in 3D cultures. This result led us to explore whether SCD1 expression and activity could impact on spheroid formation as well as ALDH1A1 activity. Using both RNA interference and pharmacological inhibition, we showed that spheroids formation was strongly impaired in the presence of SCD1-inhibiting agents. Moreover, ALDH1A1 activity, measured by flow cytometry, as well as OCT4 and Nanog mRNA levels were consistently downregulated, thus suggesting a direct role of SCD1 in the regulation of factors previously shown to be associated with cancer-initiating cells. Importantly, differentiated 2D cultures are much less sensitive to the inhibitor MF-438, underlining a specific role of SCD1 and MUFA in the maintenance and survival of CSC-like cells. In agreement with this, lipids were previously reported to regulate the self renewal of human embryonal stem cells ${ }^{34}$ and, recently, Ben-David et al. ${ }^{35}$ reported that a selective inhibitor of SCD1 was able to induce cell death in human pluripotent stem cells by inducing an ER stress response, which underlined the importance of SCD1 activity and MUFA production for these cell types.

Our data strongly support the notion that SCD1 inhibition is directly responsible for impaired survival of cancer-initiating cells, as also demonstrated by rescue experiments carried out with oleic acid. More importantly, through immunoflourescence analysis, we demonstrated that SCD1 enzymatic inhibition selectively induced apoptosis of ALDH1A1-positive cells. This was further confirmed in in vivo studies, which showed that cells derived from SCD1-inhibited spheroids give rise to smaller tumors with a strongly impaired expression of ALDH1A1. Currently, we do not know the exact mechanism through which SCD1 inhibition causes the selective death of cells with cancer-initiating properties. Mauvoisin et al. ${ }^{36}$ have recently shown in breast cancer cultures that SCD1 downregulation strongly affects oncogenic signaling through inhibition of GSK3 phosphorylation, impairment of $\beta$-catenin translocation to the nucleus, and, as a consequence, of its transactivation capacity and the expression of target genes. It will be interesting to assess whether a similar mechanism can be observed in CSC-like cells form NSCLC.

Our data strongly suggest that SCD1 may be a promising target for lung cancer. Several small molecular weight 
a

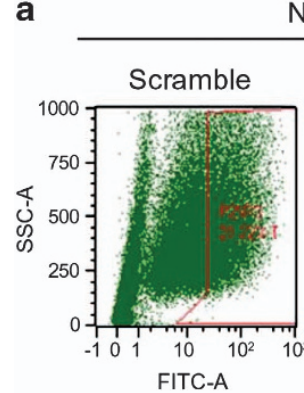

ALDH $^{\text {br }}$

21.22
$\mathrm{NCl}-\mathrm{H} 460$

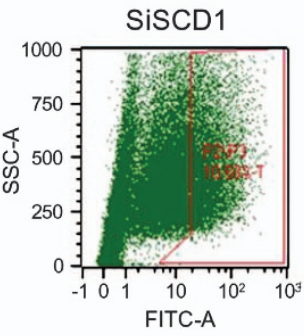

$\mathrm{ALDH}^{\mathrm{br}}$

10.68

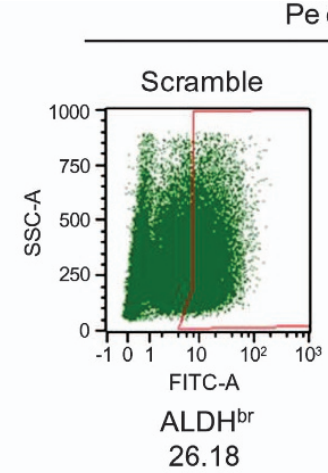

$\mathrm{Pe}$ o/11

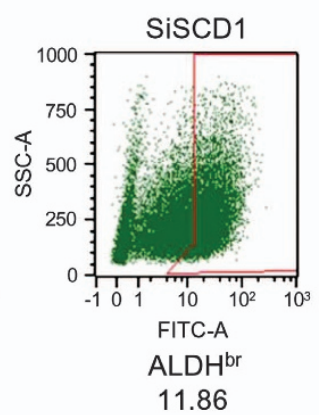

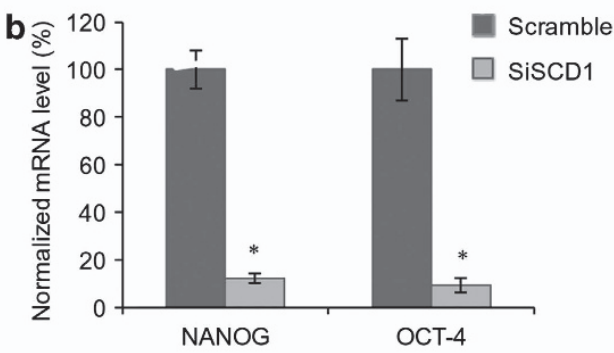

c $\mathrm{NCl}-\mathrm{H} 460$
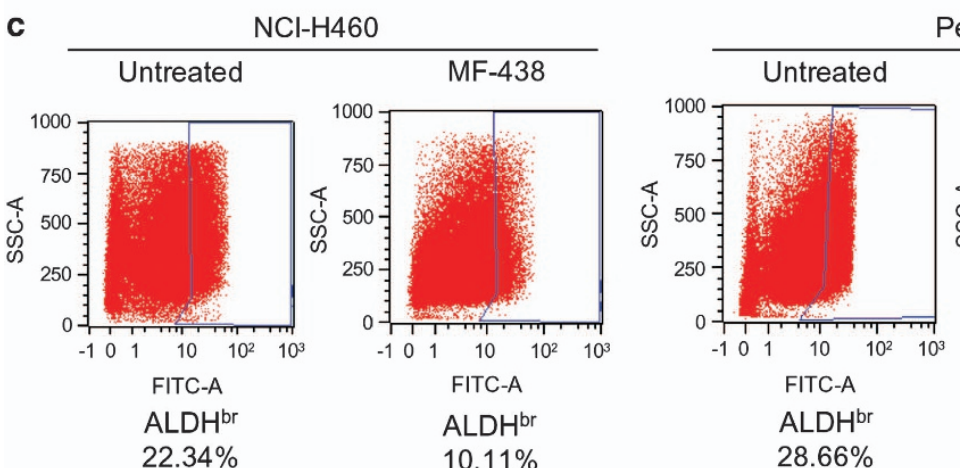

$\mathrm{Pe}$ o/11

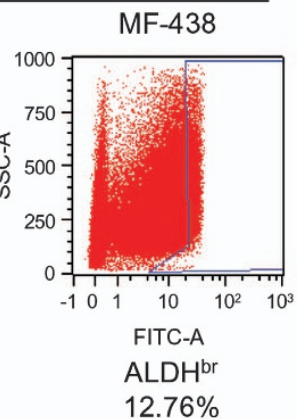

Figure 4 SCD1 inhibition reduces markers of staminality. (a) Pictures of ALDH1A1 activity determined by flow cytometry using Aldefluor assay. NCl-H460 and Pe o/11 cells were transfected with control siRNA (scramble) or a siRNA specific for SCD1 (siSCD1), cultured for $72 \mathrm{~h}$ in spheroid medium and subjected to ALDHA1A activity assay by flow cytometry. Data indicated that silencing of SCD1 reduced dramatically the percentage of cells with ALDH1A1 activity both in NCI-H460 and Pe o/11 cultures. Data are representative of three independent experiments. (b) RT-real time PCR was performed to evaluate mRNA relative expressions of OCT4 and Nanog in siSCD1 and scrambletransfected NCI-H460 cell cultures. Results demonstrated a decreased in mRNA levels of both OCT4 and Nanog genes in siSCD1-transfected cells in respect to control siRNA-transfected spheroids. Histograms show the means of at least three independent experiments. Bars indicate S.D. Student's t-test ${ }^{*} P<0.01$ in siSCD1 versus control siRNA-transfected cells. GAPDH was used as the normalization. (c) Representative pictures of flow cytometry analysis for ALDH1A1 activity. Spheroids of Pe o/11 and NCl$\mathrm{H} 460$ cell cultures treated for $72 \mathrm{~h}$ with MF-438 $(1 \mu \mathrm{M})$ reduced the percentage of positive cells with ALDH1A1 activity. Data are representative of three independent experiments

inhibitors for SCD1, such as MF-438, have been developed during the last years, which have good oral bioavailability and PK properties. ${ }^{17,18}$ Commonly observed adverse consequences of prolonged in vivo inhibition of SCD1 are skin damage with dry eye, squinting and alopecia, which are all reversible on discontinuation. These adverse effects may severely limit further development of SCD1 inhibitors for the treatment of cancer. Several approaches to limit side effects of systemic SCD1 inhibitors are under consideration, which include development of partial inhibitors or intermittent dosing. In addition, an important factor to take into account when considering SCD1 as target for cancer therapy and which emerges from our study, is that progenitor and terminally differentiated cancer cells have a low sensitivity to SCD1 inhibition as compared with CSC-like cells. Hence, SCD1 inhibitors cannot be expected to act as cancer-debulking agents but rather as selective tools to impair or delay cancer recurrence.

\section{Materials and Methods}

Cell cultures. NCI-H460 cell line was from ATCC (Manassas, VA, USA) and was cultured in RPMI-1640 medium (Sigma, St. Louis, MO, USA) supplemented with 10\% FBS (Sigma). Primary cell cultures were obtained from individual patients affected by NSCLC using a procedure previously described by Mancini et al. ${ }^{30}$ Malignant pleural fluid cells were submitted to immunocytochemistry to confirm adenocarcinoma cell origin. Diagnoses were considered 'true positives' if they were in agreement with the consensus cytological diagnosis. Experiments were approved by the Sant' Andrea Hospital Ethics Committee 2010 (504/10). All patients agreed to participate to the study and signed an informed consent form. 

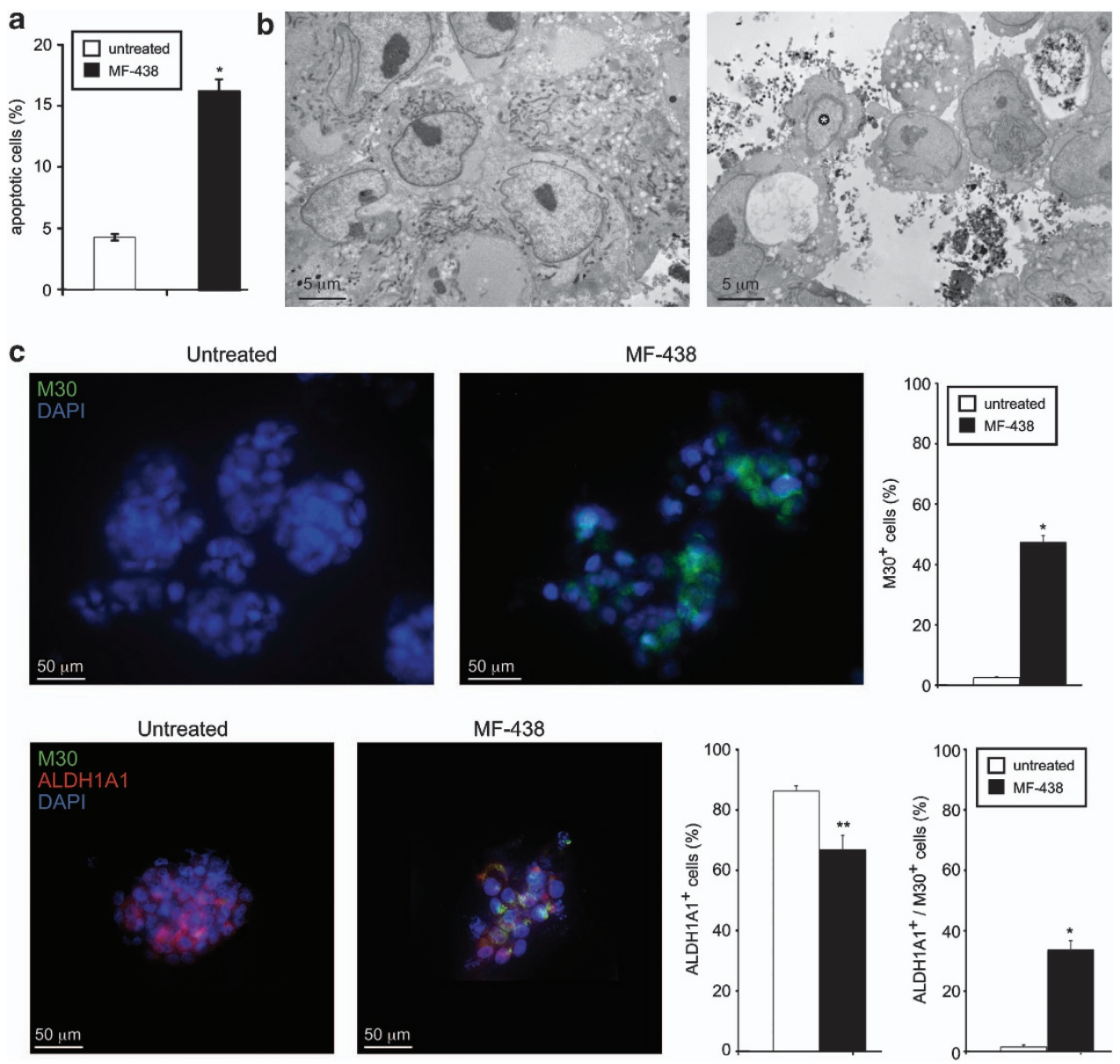

Figure 5 MF-438 induces apoptosis and cellular damage in Pe o/11 spheroids. (a) Flow cytometry analysis using Annexin $\mathrm{V}$ staining, showed apoptosis induction in Pe o/11 spheroids treated for $72 \mathrm{~h}$ with MF-438 $(1 \mu \mathrm{M})$. Data shown in the histogram are the means of three independent experiments, where bars represent S.D. ${ }^{*} P<0.01$ in treated versus untreated spheroids. (b) Analysis of ultrastructural features demonstrated that $\mathrm{Pe}$ o/11-untreated spheroids (left panel) showed nuclei generally rounded or, occasionally lobulated, with chromatin finely dispersed and prominent nucleoli. The cytoplasm displayed numerous mitochondria, rod-shaped or elongated, and variable amounts of organelles. Pe o/11-treated cells (right panel) exhibit cellular damage findings such as cytoplasmatic vacuolization, mitochondrial swelling, apoptotic nuclei with areas of marginal, dense-staining chromatin (see asterisk) and, although less frequently, nuclear and cellular fragmentation. Both spheroid cultures, undergone or not to MF-438 treatment, did not exhibit classical junctional complex but rather few continuous junctions and displayed discontinuous and short surface microvilli. (TEM, uranyl acetate/lead citrate). (c) Upper panel: Pe o/11 cultures were treated or not with MF-438 $1 \mu \mathrm{M}$ as described above and immunostained with M30 CytoDEATH Fluorescein monoclonal antibody, able to detect the caspase-cleaved cytokeratin 18. Quantitative immunofluorescence analysis indicated that the percentage of cells presenting positive M30 signal was higher in Pe o/11-treated cultures (47.3\% versus 2.4\%; $\left.{ }^{*}<0.0001\right)$. Lower panel: the co-immunostaining for M30 and the stemness marker ALDH1A1 showed that the treatment with MF-438 induced a decrease in ALDH1A1-positive cells $\left(66.8 \%\right.$ versus $86.2 \%$ obtained from untreated spheroids; Student's $t$-test: $\left.{ }^{* \star} P<0.05\right)$ and that the SCD1 inhibitor selectively killed the cells with stem-like properties, in fact most of the apoptotic M30 positive cells were also positive for ALDH1A1 (31.6\% of $\mathrm{M} 30^{+} / \mathrm{AldhA} 1^{+}$versus $3.0 \%$ of $\mathrm{M} 30^{+} / \mathrm{AldhA} 1^{-}$cells in Pe 0/11 MF-438 treated; Student's $t$-test: $\left.{ }^{*} P<0.0001\right)$. Data represent the means of three independent experiments. Bars indicate S.D.

Each distinct culture was named $\mathrm{Pe}$ (from Pleural effusion) followed by a progressive identification letter and a number indicating the year when the culture was originated. As previously reported in Mancini et al., ${ }^{30}$ to avoid long periods of adaptations in cell culture all samples were banked in liquid nitrogen at passage 3 (p3), on average 1 month following MPE collection. All further experiments described in this study were conducted with $\mathrm{p} 5$ to $\mathrm{p} 10$ cells, that is, cells in the initial passages after thawing, unless otherwise specified. Spheroids cultures were obtained from adherent culture plating single cells at clonally density in serum-free medium, as reported by Mancini et al..$^{30}$ and Giarnieri et al. ${ }^{37}$

ALDH activity assay. Second generation of spheroids was analyzed for ALDH1A1 enzymatic activity using Aldefluor kit (Stem Cell Technologies, Vancouver, BC, Canada) according to manufacturer's instructions and as previously described. ${ }^{30,31,38}$ Brightly fluorescent ALDH1A1-positive cells were detected in the green fluorescence channel, and samples treated with the specific ALDH1A1 inhibitor DEAB were used as the control to set the gates defining the
ALDH1A1-positive region, defined at $0.2 \%$. Samples were also stained with propidium iodide (Invitrogen, Carlsbad, CA, USA) to exclude nonviable cells in flow cytometric analysis. Samples were analyzed in a MACSQuant Analyzer Flow Cytometer (Miltenyi Biotec, Bergisch Gladbach, Germany).

Western blot analysis. Cell lysates obtained using RIPA buffer (Sigma) were separated on SDS/PAGE acrylamide gel and transferred overnight on nitrocellulose membranes. Membranes were blocked with $5 \%$ milk, incubated overnight with the appropriate primary antibody, followed by the secondary antibody HRP-conjugated, and developed with ECL western blotting substrate (Promega, Madison, WI, USA). The primary antibodies used were the following: anti-SCD1 (Cell Signaling Technology, Beverly, MA, USA) and anti-GAPDH (Sigma). All results were normalized over GAPDH.

Real time RT-PCR analysis. Total RNA of spheroids and adherent cells was extracted with TRIzol reagent (Life Technologies, Gaithersburg, MD, USA). 

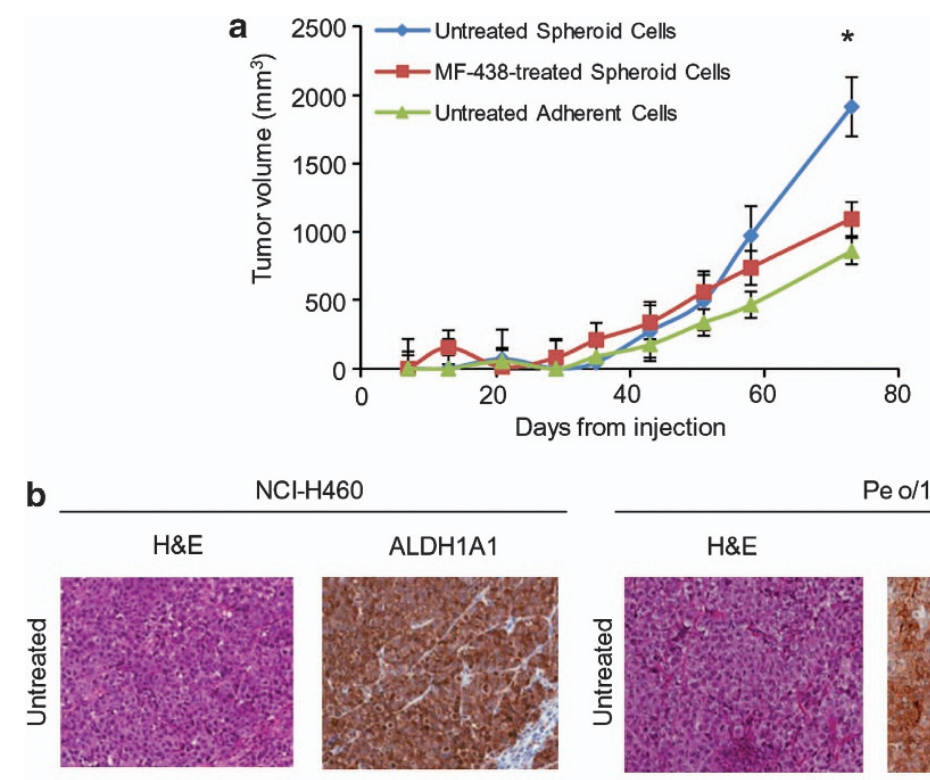

$\mathrm{NCl}-\mathrm{H} 460$

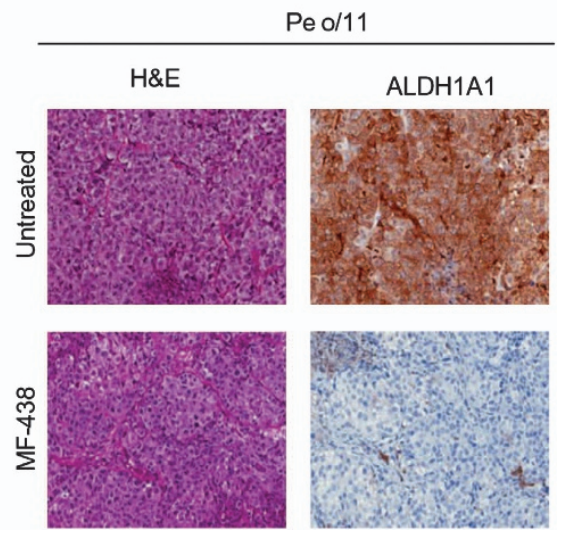

Figure 6 MF-438 impairs tumor growth and ALDH1A1 expression in vivo. (a) Single-cell suspensions were obtained from Pe o/11 adherent cells and Pe o/11 spheroids treated or not for $48 \mathrm{~h}$ with MF-438. Ten mice per group were injected subcutaneously and tumor volumes were monitored weekly. Results showed that MF-438 treatment impaired tumor growth in Pe o/11 xenografts, with a decreased volume comparable to adherent differentiated cultures. Bars indicate S.E.M. ( $n=10)$. Student's $t$-test: $P<0.05$. (b) Representative images of histology (H\&E, original magnification $\times 180$ ) and immunostaining for ALDH1A1 (original magnification $\times 180$ ) of untreated and of MF-438 treated NCl-H460 cell line (left panel) and Pe o/11 primary cell culture (right panel) injected subcutaneously in CD1 nude mice. Compared with the untreated tumors, where an intense cytoplasmic staining for ALDH1A1 was observed in all cancer cells, the MF-438-treated tumors were almost completely negative

A total of $200 \mu \mathrm{g}$ of RNA was digested with DNAse I (Invitrogen) and reversetranscribed with Superscript first-strand synthesis system (Invitrogen) according to the manufacturer's instructions. Real-time PCR was carried out with SYBR Select Master Mix (Applied Biosystems, Foster City, CA, USA). GAPDH was included for the normalization of the real time RT-PCR.

The primers used for individual genes were:

Oct4-F: 5'-GTGGAGAGCAACTCCGATG-3',

Oct4-R: 5'-TGCTCCAGCTTCTCCTTCTC-3';

Nanog-F: $5^{\prime}$-ATTCAGGACAGCCCTGATTCTTC-3',

Nanog-R: $5^{\prime}$-TTTTTGCGACACTCTTCTCTGC-3';

SCD-1-R: $5^{\prime}-$ TTCTTACACGACCACCACCA-3'

SCD-1-F: 5'-GCAGG-GGGAACCAGTATGA-3'

GAPDH-F: 5'-GGTATCGTGGAAGGACTCATGAC-3',

GAPDH-R: 5'-ATGCCAGTGAGCTTCCCGTCAGC-3'

Apoptosis assay. Analysis of apoptosis in adherent and spheroid cells was carried out by flow cytometry using Alexa fluor 488-Annexin V (Invitrogen). Second generation of spheroid cultures were treated with or without MF-438 $(1 \mu \mathrm{M})$ for $72 \mathrm{~h}$. Treated cells were collected and incubated with Annexin V for $15 \mathrm{~min}$ in the dark according to the recommendation of the manufacturer. Samples were analyzed using MacsQuant Flow Cytometer (Miltenyi Biotec)

MTT assay. Cellular growth in the presence or absence of MF-438 was evaluated using MTT assay. Adherent cultures were plated in 96 well/plate and after $24 \mathrm{~h}$ were treated with the indicate concentration of MF-438. After $72 \mathrm{~h}$ of treatment, MTT (Sigma) was added to the wells and DMSO (Sigma) was used to solubilize the MTT-formazan product. Absorbance was measured at $570 \mathrm{~nm}$ using the Multiskan EX Microplate Photometer (Thermo Scientific Dharmacon, Lafayette, CO, USA). The dose-response curves could be plotted with $50 \%$ inhibitory concentration $\left(\mathrm{IC}_{50}\right)$, and were defined with KaleidaGraph software.
Spheroid-forming assay. Cells were plated as single cells in ultralow attachment plates (Corning Incorporated, Corning, NY, USA) and were grown in sphere medium with $0.8 \%$ methylcellulose (Sigma) with or without MF-438 inhibitor at the indicated concentrations for 7-8 days. For rescue experiments, Oleic AcidBSA (Sigma) was used at $1 \mu \mathrm{M}$. We performed spheroid formation assay with second generation of spheroids plated at 1500-2000 cells/well in 96-well plates. The number of formed spheroids was counted under an inverted microscope.

siRNA transfection. Small interfering RNA-targeting SCD-1 (Sigma) or control siRNA-A (sc-37007; Santa Cruz, CA, USA) was transfected into adherent cells using Lipofectamine RNAiMAX Reagents (Invitrogen), according to the manufacturer's recommendation. After $48 \mathrm{~h}$ from transfection, cultures were grown in sphere medium and allowed to form spheroids.

Chemicals. MF-438 SCD-1 inhibitor was kindly provided by Merck (Darmstadt, Germany). A939572 was purchased from Biovision (Milpitas, CA, USA). Oleic acid-BSA was from Sigma-Aldrich (Milan, Italy).

Morphometric analysis. For the 3D morphological analysis of tumor spheroids, samples were examined under an Axiovert 200 inverted microscope (Zeiss, Oberkochen, Germany) equipped with differential interference contrast optics. For quantitative image analysis, digital micrographs of at least 200 multicellular structures and single cells for each condition were randomly captured from three different experiments using an AxioCam MRm CCD camera (Zeiss).

Projected area $(A)$, perimeter $(P)$ and two orthogonal diameters $(a$ and $b)$ were measured for each 3D structures using the Axiovision software (Zeiss). Sphericity, volume and size was subsequently calculated according to the methods proposed by Kelm et al. ${ }^{39}$ and Vinci et al. ${ }^{40}$

The sphericity of each structure was expressed calculating the Shape Factor: $\Phi=\frac{\pi \sqrt{\frac{4 A}{\pi}}}{P}$. 
The volume $\left(\mu \mathrm{m}^{3}\right)$ was corrected for Shape Factor and calculated applying: $V=\Phi \frac{4 \pi}{3}\left(\frac{P}{2 \pi}\right)^{3}$.

The size $(\mu \mathrm{m})$ was determined calculating the geometric mean diameter: $D_{\mathrm{G}}=\sqrt{a b}$.

All 3D structures were also categorized according to their morphology and classified as follows: tight spheroids (densely packed spheres with almost indiscernible individual cells), irregular aggregates (two or more cells organized in loose or compact aggregates that do not form the typical spheroid structure) and single floating cells.

Transmission electron microscopy. Cultures, treated or not with MF-438 $1 \mu \mathrm{M}$ as above, were washed three times in PBS and fixed with $2 \%$ glutaraldehyde in PBS for $2 \mathrm{~h}$ at $4{ }^{\circ} \mathrm{C}$. Samples were postfixed with $1 \%$ osmium tetroxide in veronal acetate buffer (pH 7.4) for $1 \mathrm{~h}$ at $25^{\circ} \mathrm{C}$, stained with uranyl acetate $(5 \mathrm{mg} / \mathrm{ml})$ for $1 \mathrm{~h}$ at $25^{\circ} \mathrm{C}$, dehydrated in acetone and embedded in Epon 812 (EMbed 812, Electron Microscopy Science, Hatfield, PA, USA). Ultrathin sections were examined unstained or poststained with uranyl acetate and lead hydroxide, under a Morgagni 268D transmission electron microscope (FEl, Hillsboro, OR, USA) equipped with a Mega View II charge-coupled device camera (SIS, Soft Imaging System GmbH, Munster, Germany) and analyzed with AnalySIS software (SIS).

Immunofluorescence. Cultures, untreated or treated with MF-438 $1 \mu \mathrm{M}$ as above, were washed in PBS and fixed with methanol for $4 \mathrm{~min}$ at $-20^{\circ} \mathrm{C}$. To detect the apoptotic cells, spheroids were labeled with M30 CytoDEATH Fluorescein monoclonal antibody (1:150 in PBS, Roche, Mannheim, Germany). To verify the apoptotic versus the stemness phenotype, spheroids were double labeled with M30 CytoDEATH Fluorescein and with anti-ALDH1A1 primary rabbit monoclonal antibody (1:50 in PBS) followed by goat anti-rabbit IgG-Texas Red (1:200 in PBS; Jackson Immunoresearch Laboratories, West Grove, PA, USA). Nuclei were visualized using 4,6-diamido-2-phenylindole dihydrochloride (DAPI) (1:10000 in PBS, Sigma Chemical Co., St. Louis, MO, USA). Fluorescence signals were analyzed by conventional fluorescence or by scanning in series of $0.5-\mu \mathrm{m}$ sequential optical sections with an ApoTome System (Zeiss) connected with an Axiovert 200 inverted microscope (Zeiss); image analysis and 3D reconstruction of the optical sections was then performed using the Axiovision software (Zeiss). The percentage of $\mathrm{M} 30$ and/or AldhA1-positive cells was analyzed counting for each treatment a total of 500 cells, observed in ten microscopic fields randomly taken from three different experiments.

In vivo studies. For xenograft assay, single-cell suspension was obtained by enzymatic digestion of the spheroids using different cell numbers in a volume of $100 \mu \mathrm{l}$ of a $50 \%$ GFR Matrigel (BD Biosciences, Franklin Lakes, NJ, USA). In particular, $\mathrm{Pe} 0 / 11$ and $\mathrm{NCl}-\mathrm{H} 460$ spheroids generated for $48 \mathrm{~h}$ in the absence or in the presence of SCD1 inhibitor $(500 \mathrm{nM})$ were injected subcutaneously into the right flank of ten CD1 nude mice for each group. $1 \times 10^{4}$ viable single cells for $\mathrm{NCl}-\mathrm{H} 460$ and $1 \times 10^{5}$ viable single cells for $\mathrm{Pe} 0 / 11$, respectively, were used. Tumors were measured with a caliper at weekly intervals and tumor volumes were calculated using the formula: (length $\times$ width $\left.^{2}\right) / 2$. After $\sim 70$ days from injection, mice were killed and tumors excised for immunohistochemistry analysis.

All studies have been performed in accordance with 'Directive 86/609/EEC on the protection of Animals used for Experimental and other scientific purposes and made effective in Italy by the Legislative Decree DL $116 / 92$ as described in Ricci et al. ${ }^{31} \mathrm{All}$ animal protocols used for this study were reviewed and approved by Takis Ethical Committee (TKEC-2012-02).

Immunohistochemistry. Paraffin sections of tumors generated subcutaneously in CD1 nude mice after injection of NCl-H460 cell line or of $\mathrm{Pe} 0 / 11$ primary cell culture were immunostained for ALDH1A1 (clone EP1933Y, Epitomics, Burlingame, CA, USA) using an automated immunostainer (DAKO, Glostrup, Denmark).

Statistical methods. For almost all experiments, statistics were performed using two-tailed Student's $t$-test. All values shown in the text and figures are \pm S.D., where $n=3$. $P$-values $<0.05$ were assumed as statistically significant.

For the morphometric analysis, to compare variables that do not assume Gaussian distribution, Mann-Whitney non-parametric test was used. Data are presented with the Tukey box-and-whisker plot, where the central box represents the interquartile ranges (IR; 25 th to 75 th percentile), the middle line represents the median, and the horizontal lines represent the minimum and the maximum value of observation range. Values are expressed as median $\pm I R$. To compare variables that assume normal distribution, Student's t-test was used and values are expressed as mean \pm S.E. from three independent experiments. Chi-square test was used to compare categorical variables. $P$-values $<0.05$ were assumed as statistically significant.

For the in vivo studies using $\mathrm{NCl}-\mathrm{H} 460$ cell line, statistical analysis was performed using Fisher's exact test.

\section{Conflict of Interest}

The authors declare no conflict of interest.

Acknowledgements. This work was supported in part by AIRC grant IG10334 and by MIUR PRIN 2009 grant to $G$ Ciliberto. This work has also been supported in part by FILAS grant to R Mancini, FILAS-CR-2011-1184. We are grateful to Merck for making available the SCD1 inhibitor MF-438. We also thank Dott Roberta Barzellotti for technical support.

1. Menendez JA, Lupu R. Fatty acid synthase and the lipogenic phenotype in cancer pathogenesis. Nat Rev Cancer 2007; 7: 763-777.

2. Igal RA. Stearoyl-CoA desaturase-1: a novel key player in the mechanisms of cell proliferation, programmed cell death and transformation to cancer. Carcinogenesis 2010; 31: $1509-1515$.

3. Ntambi JM. Regulation of stearoyl-CoA desaturase by polyunsaturated fatty acids and cholesterol. J Lipid Res 1999; 40: 1549-1558.

4. Riboni L, Ghidoni R, Sonnino S, Omodeo-Salè F, Gaini SM, Berra B. Phospholipid content and composition of human meningiomas. Neurochem Pathol 1984; 2: 171-188.

5. Zhang L, Ge L, Parimoo S, Stenn K, Prouty SM. Human stearoyl-CoA desaturase: alternative transcripts generated from a single gene by usage of tandem polyadenylation sites. Biochem J 1999; 340: 255-264.

6. Beiraghi S, Zhou M, Talmadge CB, Went-Sumegi N, Davis JR, Huang D et al. Identification and characterization of a novel gene disrupted by a pericentric inversion inv(4) (p13.1q21.1) in a family with cleft lip. Gene 2003; 309: 11-21.

7. Robert J, Montaudon D, Hugues P. Incorporation and metabolism of exogenous fatty acids by cultured normal and tumoral glial cells. Biochim Biophys Acta 1983; 752: 383-395

8. Scaglia N, Chisholm JW, Igal RA. Inhibition of stearoylCoA desaturase-1 inactivates acetylCoA carboxylase and impairs proliferation in cancer cells: role of AMPK. PLoS One 2009; 4: e6812.

9. Mason P, Liang B, Li L, Fremgen T, Murphy E, Quinn A et al. SCD1 inhibition causes cancer cell death by depleting mono-unsaturated fatty acids. PLoS One 2012; 7: e33823.

10. Roongta UV, Pabalan JG, Wang X, Ryseck RP, Fargnoli J, Henley BJ et al. Cancer cell dependence on unsaturated fatty acids implicates stearoyl-CoA desaturase as a target for cancer therapy. Mol Cancer Res 2011; 9: 1551-1561.

11. Hess D, Igal RA. Genistein downregulates de novo lipid synthesis and impairs cell proliferation in human lung cancer cells. Exp Biol Med (Maywood) 2011; 236: 707-713.

12. Du X, Wang QR, Chan E, Merchant M, Liu J, French D et al. FGFR3 stimulates stearoyl CoA desaturase 1 activity to promote bladder tumor growth. Cancer Res 2012; 72: 5843-5855.

13. Fritz V, Benfodda Z, Rodier G, Henriquet C, Iborra F, Avancès $C$ et al. Abrogation of de novo lipogenesis by stearoyl-CoA desaturase 1 inhibition interferes with oncogenic signaling and blocks prostate cancer progression in mice. Mol Cancer Ther 2010; 9: 1740-1754.

14. Luyimbazi D, Akcakanat A, McAuliffe PF, Zhang L, Singh G, Gonzalez-Angulo AM et al. Rapamycin regulates stearoyl $\mathrm{CoA}$ desaturase 1 expression in breast cancer. Mol Cancer Ther 2010; 9: 2770-2784.

15. Scaglia N, Igal RA. Inhibition of Stearoyl-CoA Desaturase 1 expression in human lung adenocarcinoma cells impairs tumorigenesis. Int J Oncol 2008; 33: 839-850.

16. von Roemeling CA, Marlow LA, Wei JJ, Cooper SJ, Caulfield TR, Wu K et al. Stearoyl-CoA desaturase 1 is a novel molecular therapeutic target for clear cell renal cell carcinoma. Clin Cancer Res 2013; 19: 2368-2380.

17. Isabel E, Powell DA, Black WC, Chan CC, Crane S, Gordon R et al. Biological activity and preclinical efficacy of azetidinyl pyridazines as potent systemically-distributed stearoyl-CoA desaturase inhibitors. Bioorg Med Chem Lett 2011; 21: 479-483.

18. Léger S, Black WC, Deschenes D, Dolman S, Falgueyret JP, Gagnon M et al. Synthesis and biological activity of a potent and orally bioavailable SCD inhibitor (MF-438). Bioorg Med Chem Lett 2010; 20: 499-502.

19. Nguyen LV, Vanner R, Dirks P, Eaves CJ. Cancer stem cells: an evolving concept. Nat Rev Cancer 2012; 12: 133-143.

20. Conway AE, Lindgren A, Galic Z, Pyle AD, Wu H, Zack JA et al. A self-renewal program controls the expansion of genetically unstable cancer stem cells in pluripotent stem cellderived tumors. Stem Cells 2009; 27: 18-28. 
21. Eramo A, Lotti F, Sette G, Pilozzi E, Biffoni M, Di Virgilio A et al. Identification and expansion of the tumorigenic lung cancer stem cell population. Cell Death Differ 2008; 15 : 504-514.

22. Dontu G, Wicha MS. Survival of mammary stem cells in suspension culture: implications for stem cell biology and neoplasia. J Mammary Gland Biol Neoplasia 2005; 10: 75-86.

23. Akunuru S, James Zhai Q, Zheng Y. Non-small cell lung cancer stem/progenitor cells are enriched in multiple distinct phenotypic subpopulations and exhibit plasticity. Cell Death Dis 2012; 3: e352.

24. Bartucci M, Svensson S, Romania P, Dattilo R, Patrizii M, Signore M et al. Therapeutic targeting of Chk1 in NSCLC stem cells during chemotherapy. Cell Death Differ 2012; 19: 768-778.

25. Abdullah LN, Chow EK. Mechanisms of chemoresistance in cancer stem cells. Clin Trans Med 2013; 2: 1326-2-3.

26. Deng S, Yang X, Lassus H, Liang S, Kaur S, Ye Q et al. Distinct expression levels and patterns of stem cell marker, aldehyde dehydrogenase isoform 1 (ALDH1), in human epithelial cancers. PLoS One 2010; 5: e10277.

27. Sullivan JP, Spinola M, Dodge M, Raso MG, Behrens C, Gao B et al. Aldehyde dehydrogenase activity selects for lung adenocarcinoma stem cells dependent on notch signaling. Cancer Res 2010; 70: 9937-9948.

28. Ucar D, Cogle CR, Zucali JR, Ostmark B, Scott EW, Zori R et al. Aldehyde dehydrogenase activity as a functional marker for lung cancer. Chem Biol Interact 2009; 178 48-55.

29. Moreb JS. Aldehyde dehydrogenase as a marker for stem cells. Curr Stem Cell Res The 2008; 3: 237-246.

30. Mancini R, Giarnieri E, De Vitis C, Malanga D, Roscilli G, Noto A et al. Spheres derived from lung adenocarcinoma pleural effusions: molecular characterization and tumor engraftment. PLoS One 2011; 6: e21320.

31. Ricci A, De Vitis C, Noto A, Fattore L, Mariotta S, Cherubini E et al. TrkB is responsible for EMT transition in malignant pleural effusions derived cultures from adenocarcinoma of the lung. Cell Cycle 2013; 12: 1696-1703.
32. Herbst RS, Heymach JV, Lippman SM. N Engl J Med. Lung Cancer 2008; 359: $1367-1380$.

33. Mani SA, Guo W, Liao MJ, Eaton EN, Ayyanan A, Zhou AY et al. The epithelial-mesenchymal transition generates cells with properties of stem cells. Cell 2008; 133: 704-715.

34. Garcia-Gonzalo FR, Izpisúa Belmonte JC. Albumin-associated lipids regulate human embryonic stem cell self-renewal. PLoS One 2008; 3: e1384.

35. Ben-David U, Gan QF, Golan-Lev T, Arora P, Yanuka O, Oren YS et al. Selective elimination of human pluripotent stem cells by an oleate synthesis inhibitor discovered in a high-throughput screen. Cell Stem Cell 2013; 12: 167-167.

36. Mauvoisin D, Charfi C, Lounis AM, Rassart E, Mounier C. Decreasing stearoyl-CoA desaturase- 1 expression inhibits $\beta$-catenin signaling in breast cancer cells. Cancer $\mathrm{SCi}$ 2013; 104: 36-42.

37. Giarnieri E, De Vitis C, Noto A, Roscilli G, Salerno G, Mariotta S et al. EMT markers in lung adenocarcinoma pleural effusion spheroid cells. J Cell Physiol 2013; 228: 8.

38. Li T, Su Y, Mei Y, Leng Q, Leng B, Liu Z et al. ALDH1A1 is a marker for malignant prostate stem cells and predictor of prostate cancer patients' outcome. Lab Invest 2010; 90: 234-244.

39. Kelm JM, Timmins NE, Brown CJ, Fussenegger M, Nielsen LK. Method for generation of homogeneous multicellular tumor spheroids applicable to a wide variety of cell types. Biotechnol Bioeng 2003; 83: 173-180.

40. Vinci M, Gowan S, Boxall F, Patterson L, Zimmermann M, Court W et al. Advances in establishment and analysis of three-dimensional tumor spheroid-based functional assays for target validation and drug evaluation. BMC Biol 2012 10-29.

(c) (i) $\ominus$ Cell Death and Disease is an open-access journal published by Nature Publishing Group. This work is licensed under a Creative Commons Attribution-NonCommercialNoDerivs 3.0 Unported License. To view a copy of this license, visit http://creativecommons.org/licenses/by-nc-nd/3.0/

Supplementary Information accompanies this paper on Cell Death and Disease website (http://www.nature.com/cddis) 